\title{
Three-Way Crossover Network by Using Bernstein Polynomial
}

\author{
V. Chutchavong, S. Poungpayom, O. Sangaroon \\ Faculty of Engineering \\ King Mongkut's Institute of Technology Ladkrabang \\ Bangkok, Thailand \\ kcvanvis@kmitl.ac.th
}

\author{
K. Janchitrapongvej \\ Faculty of Science and Technology \\ Southeast Bangkok College \\ Bangkok, Thailand \\ kjkanok@gmail.com
}

\begin{abstract}
This paper presents the design of the three-way crossover network by using Bernstein polynomial. The Bernstein polynomial is applied to the Bernstein filter which has the best characteristics with more advantages. For example, it has a maximally flat magnitude, a linear-phase and a maximally flat delay with parameters of $n, K$ and $\varepsilon$. For this reason, the three-way crossover network is designed by Bernstein filter. Moreover, the analog filter is converted into the digital filter by using the bilinear transformation. Both analog filter and digital filter have better performance for designing the three-way crossover network. In addition, the experimental results at the real time of the three-way crossover network can realize via FiO Board and RapidSTM32.
\end{abstract}

Keywords-Bernstein Polynomials; Bilinear Transform; Crossover Network; Filter

\section{INTRODUCTION}

The audio frequency is a rate of oscillation in the range of about $20 \mathrm{~Hz}$ to $20 \mathrm{kHz}$ which is also used in the loudspeaker system. However, in practice, the single loudspeaker cannot respond the frequency requirements in the whole audio frequency. For this reason, the loudspeaker system has to solve the problem by using crossover network [1-2]. The crossover networks have two types; the two-way crossover network and the three-way crossover network. The three-way crossover network is composed of the high pass filter, the band pass filter, and the low pass filter [3-4]. The important condition of the crossover network must be required as follows; the summation of magnitude response is flat or constant, the phase is linear, and the group delay is flat [3-5]. In this paper, the Bernstein polynomial is applied to the Bernstein filter. As it is known that the Bernstein filter has flexible parameters to adjust its performance for the best results. For example, firstly, it has the maximally flat (MAXFLAT) magnitude in both the pass-band and the stopband. Secondly, the attenuation in the stop-band can be changed with parameters of $\varepsilon$. Thirdly, it has very linearphase and can be changed the slope of phase with parameters of $K$ and $\varepsilon$. Next, the amount of phase and delay using $K$ and $\varepsilon$ can be varied. Finally, it has a maximally-flat delay which is very important for designing of the filter [6]. Therefore, the proposed three-way crossover network selects the Bernstein filter for designing and realizing the efficiency of the crossover network.
The rest of the paper is organized as follows. In Section 2, we described the Bernstein filter. The design of the threeway crossover network using Bernstein polynomial and the simulation results are presented in Section 3. Then, Section 4 shows the experimental results of the proposed three-way crossover network. Finally, the conclusion is given in Section 5.

\section{BERNSTEIN FILTER}

The Bernstein filter which was designed from the Bernstein polynomial corresponding to the desired function $(f(x))$. Let $f(x)$ is a function defined on the interval $(0,1)$. The $\mathrm{n}^{\text {th }}$ Bernstein polynomial is given by [6-8]

$$
B_{n}(f ; x)=\sum_{i=0}^{n} f\left(\frac{i}{n}\right)\left(\begin{array}{l}
n \\
i
\end{array}\right) x^{i}(1-x)^{n-i}
$$

$$
\text { For } i=0,1, \ldots, n \text { where }\left(\begin{array}{l}
n \\
i
\end{array}\right)=\frac{n !}{i !(n-i) !} \text {. Considering } f(x)
$$

is a low pass function, as in (2), to analyze the Bernstein filter. The function $f\left(\frac{i}{n}\right)$ can be defined as

$$
f\left(\frac{i}{n}\right)= \begin{cases}1, & 0 \leq i \leq n-K \\ 0, & n-K+1 \leq i \leq n\end{cases}
$$

where $K$ is the number of successive discrete points at the zero values function. 


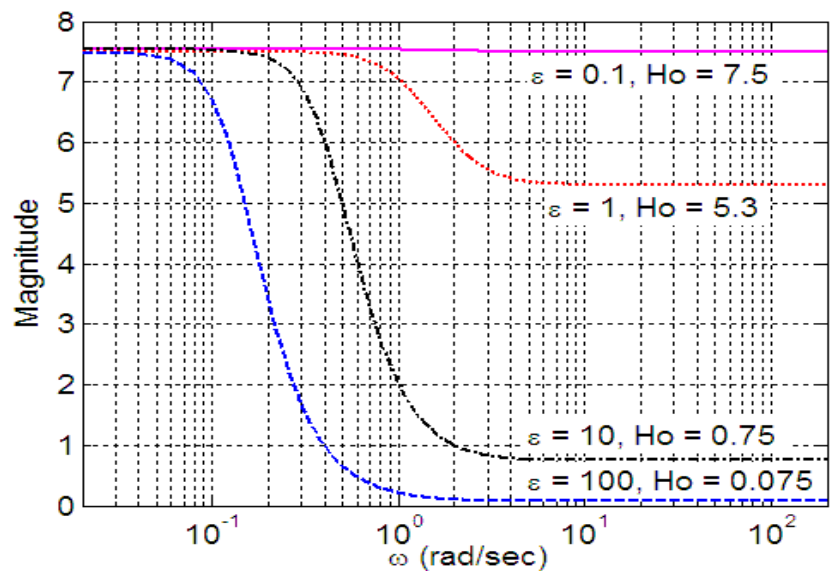

Figure 1. The magnitude characteristics.

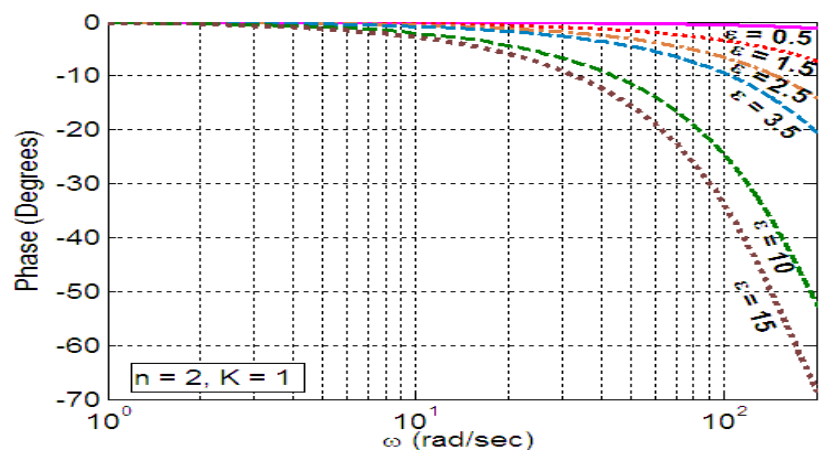

Figure 2. The phase characteristics.

Let us assume the parameters of the low pass transfer function of the $4^{\text {th }}$ Bernstein filter are $n=2$ and $k=1$. On design of the Bernstein filter can be referred to [6][8].

Fig. 1 shows the comparison of magnitude characteristics. It can be concluded that the transfer functions have MAXFLAT magnitude in the pass-band. In addition, the attenuation in the stop-band can be changed depending on $\varepsilon$. Hence, the Bernstein filter can be applied in control systems such as the compensating circuits. Fig. 2 shows the comparison of phase characteristics. It can be noted that the phase characteristics are very linear. Moreover, the Bernstein filter can change the phase slope depending on $\varepsilon$.

\section{The Design of ThreE-WAY CROSSOVER NeTWORK USING BERNSTEIN POLYNOMIAL}

\section{A. The Design of the Three-way Crossover Network}

The Three-way crossover network is designed by using the Bernstein polynomial. Firstly, beginning with the all pass function by choosing $n=1, k=1, \varepsilon=0$ and $f(x)=1$ and the substituting into (1) which, can be obtained by

$$
|D(s)|^{2}=\left(1+\varepsilon^{2}\right) s^{4}-2 s^{2}+1
$$

Thus, the all pass transfer function of the $2^{\text {nd }}$ Bernstein filter is obtained by

$$
H_{A P}(s)=\frac{s^{2}-2 s+1}{s^{2}+2 s+1}
$$

Secondly, the conjugate is used for designing the all pass transfer function of the $4^{\text {th }}$ Bernstein filter, and defined as

$$
H_{A P}(s)=\frac{s^{4}-2 s^{2}+1}{s^{4}+4 s^{3}+6 s^{2}+4 s+1}
$$

Thirdly, the new idea of the design of the three-way crossover network is

$$
H_{A P}(s)=H_{H P}(s)+H_{B P}(s)+H_{L P}(s)
$$

So that, the high pass transfer function $H_{H P}(s)$, the band pass transfer function $H_{B P}(s)$ and the low pass transfer function $H_{L P}(s)$ can be realized from (5) according to the following

$$
\begin{aligned}
& H_{H P}(s)=\frac{s^{4}}{s^{4}+4 s^{3}+6 s^{2}+4 s+1} \\
& H_{B P}(s)=\frac{-2 s^{2}}{s^{4}+4 s^{3}+6 s^{2}+4 s+1} \\
& H_{L P}(s)=\frac{1}{s^{4}+4 s^{3}+6 s^{2}+4 s+1}
\end{aligned}
$$

where the magnitude responses of the three-way crossover network is shown in Fig. 3.

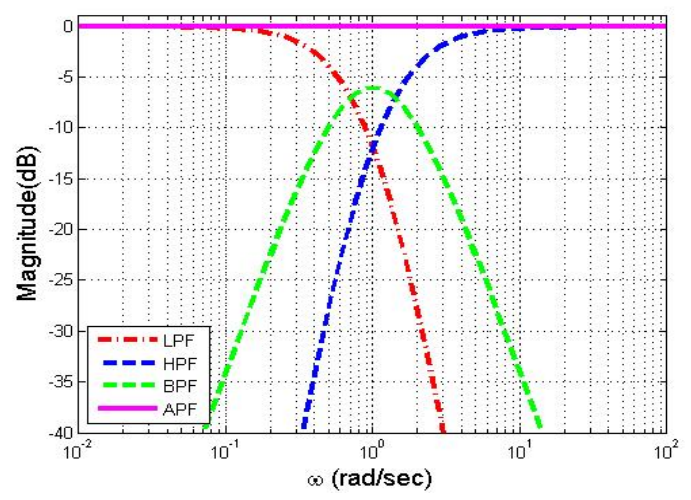

Figure 3. The magnitude responses of the three-way crossover network by using Bernstein polynomial. 


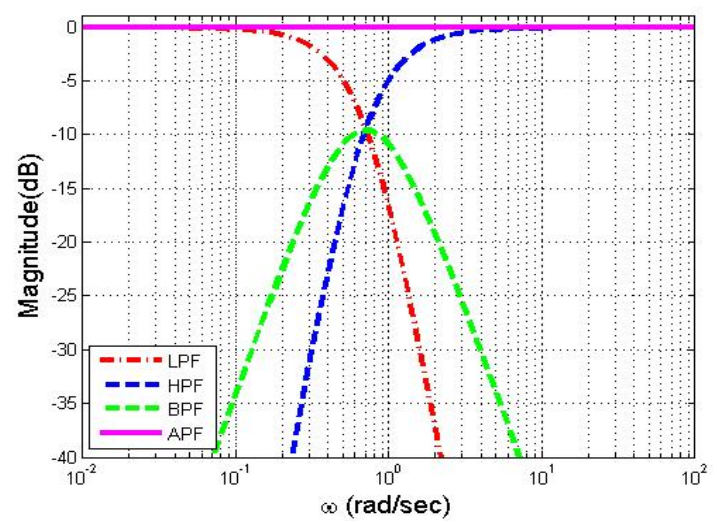

Figure 4. The magnitude responses of the three-way crossover network by using Bernstein polynomial with $\varepsilon=1.7$.

Fig. 3 shows the magnitude response of the three-way crossover network. As observed from Fig. 3, the proposed crossover network displays a better performance indicates that the sum of magnitude responses is constant or flat. In addition, the phase response is zero. Moreover, the proposed crossover network can be changed the slope in the transition band and the cross frequency by depending on $\varepsilon$ as shown in Fig. 4.

Fig. 4 shown the magnitude response of the three-way crossover network by using Bernstein polynomial with $\varepsilon=1.7$. Then, the transfer functions of all filter types can be shown as

$$
\begin{aligned}
& H_{A P}(s)=\frac{s^{4}-0.5002 s^{2}+0.2502}{s^{4}+2.4500 s^{3}+2.5010 s^{2}+1.2255 s+0.2502} \\
& H_{H P}(s)=\frac{s^{4}}{s^{4}+2.4500 s^{3}+2.5010 s^{2}+1.2255 s+0.2502} \\
& H_{B P}(s)=\frac{-0.5002 s^{2}}{s^{4}+2.4500 s^{3}+2.5010 s^{2}+1.2255 s+0.2502} \\
& H_{L P}(s)=\frac{0.2502}{s^{4}+2.4500 s^{3}+2.5010 s^{2}+1.2255 s+0.2502}
\end{aligned}
$$

To sum up, the Bernstein filter has the best characteristics with more advantages among the Butterworth filter, the Chebyshev filter, and the Bessel-Thomson filter. Therefore in this paper, the Bernstein polynomial is used for proving the efficiency in designing the three-way crossover network.

\section{B. Simulation Results}

The development of a digital three-way crossover network is to convert an analog transfer function to a digital transfer function based on bilinear transformation [9]. The bilinear transformation is given by

$$
H(z)=\left.H(s)\right|_{S=\frac{2}{T}}\left(\frac{1-z^{-1}}{1+z^{-1}}\right)
$$

Thus the desired transfer functions are given by

$$
\begin{aligned}
& H_{H P}(z)=\frac{z^{4}-4 z^{3}+6 z^{2}-4 z+1}{15.6128 z^{4}-0.3082 z^{3}+0.0782 z^{2}-0.0006 z+0.0002} \\
& H_{B P}(z)=\frac{-z^{4}+2 z^{2}-1}{8.1186 z^{4}-0.1623 z^{3}+0.0407 z^{2}-0.0003 z+0.0001} \\
& H_{L P}(z)=\frac{z^{4}+4 z^{3}+6 z^{2}+4 z+1}{16.2397 z^{4}-0.3206 z^{3}+0.0813 z^{2}-0.0006 z+0.0002}
\end{aligned}
$$

Fig. 5 shows the magnitude response of the digital threeway crossover network based on bilinear transformation. It can be noted that the magnitude summation is flat. Moreover, it is realized by the MATLAB Simulink as illustrated in Fig. 6.

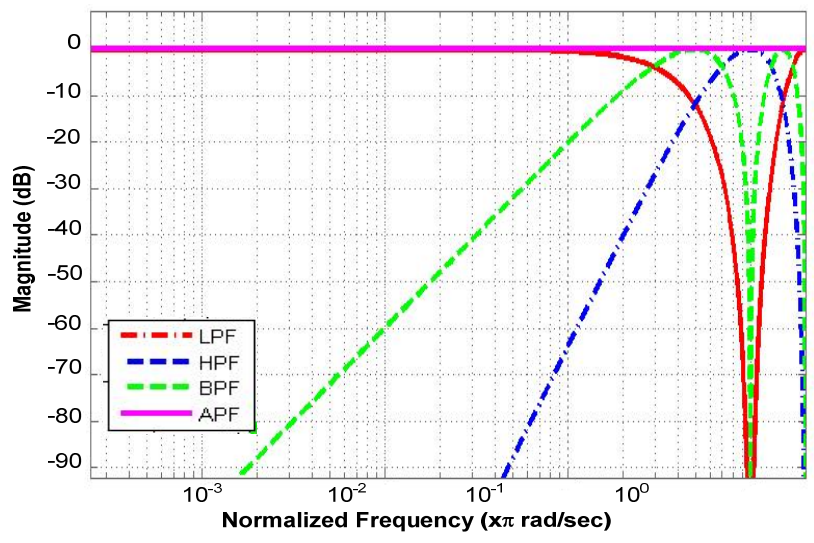

Figure 5. The magnitude responses of the digital three-way crossover network.

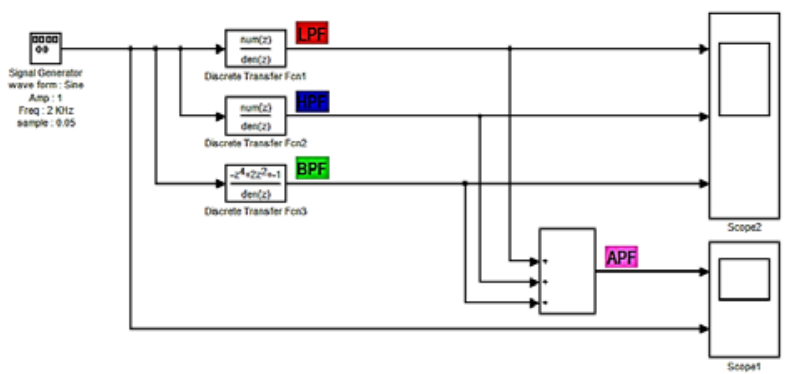

Figure 6. The block diagram of the simulation of the three-way crossover network by using the MATLAB Simulink.

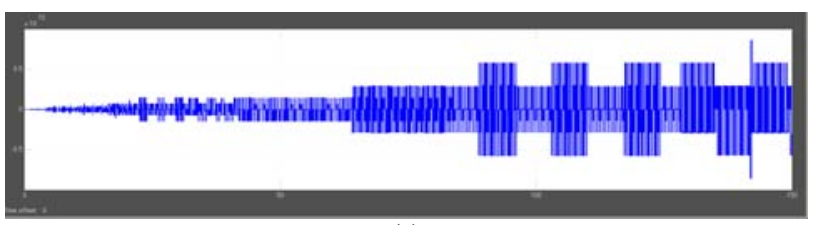

(a) 


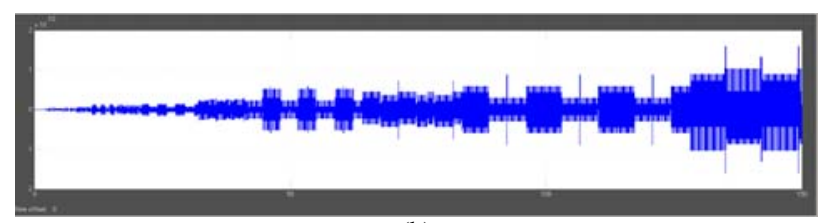

(b)

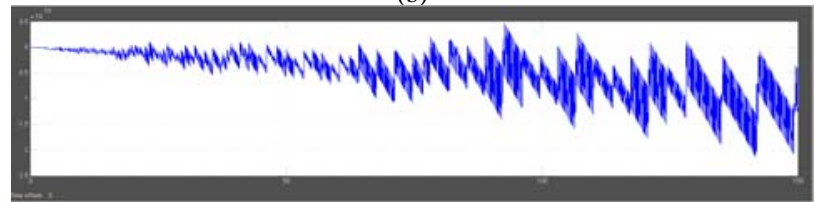

(c)

Figure 7. The simulation results of the digital three-way crossover network.

The simulation results of the digital three-way crossover network are shown in Fig. 7. The results are composed of the high pass transfer function, the band pass transfer function and the low pass transfer function as shown in Fig. 7(a), Fig. 7(b) and Fig. 7(c), respectively.

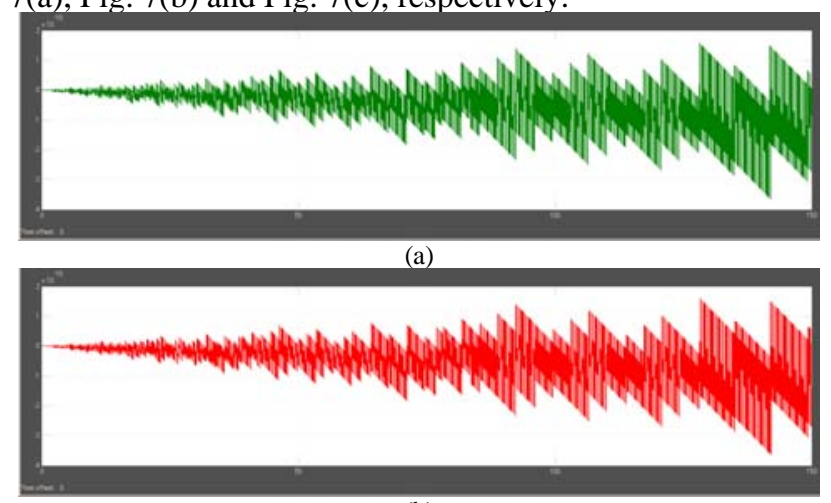

(b)

Figure 8. The comparison between the input signal and output signal of the digital three-way crossover network.

Fig. 8 shows the comparison between the input signal with a $2 \mathrm{~Hz}$ sine wave and the output signal of the all pass transfer function as illustrated in Fig. 8(a) and Fig.8(b), respectively. As a consequence, the digital three-way crossover network by using Bernstein polynomial is a good method with the best magnitude and phase responses. It is also proved to be efficient in the digital audio signal.

\section{EXPERIMENTAL RESULTS}

A real-time realization of the three-way crossover network is implemented via the FiO Board and RapidSTM32 Blockset. In this experiment, the input signal was generated by the sweep generator and the oscilloscope is used to display the output signal as shown in Fig. 9. Fig. 10 shows the experimental result of the three-way crossover network. In the oscilloscope, the yellow line (the upper graph) displays the input signal and the blue line (the lower graph) displays the output signal. As the experimental result, the proposed three-way crossover network gives a good performance. Furthermore, the simulation and experimental results are in good agreement in all types of filters.
Nevertheless, since the page is limited, the comparison results of all pass filters are shown in Fig. 10.

\section{CONCLUSION}

The design of the three-way crossover network by using Bernstein polynomial was presented in order to improve the performance of the audio signal in the loudspeaker system. The Bernstein filter is the best characteristics; the MAXFLAT magnitude, a linear phase and a constant delay. Additionally, these characteristics can be changed by depending on $\varepsilon$. The three-way crossover network can prove the necessary conditions of the analog crossover network. As the simulation results, the magnitude summation is flat or constant and the phase is zero. Moreover, the threeway crossover network is implemented via the FiO Board and RapidSTM32 Blockset. As the experimental results, the proposed three-way crossover network is also flat and an efficient implementation.

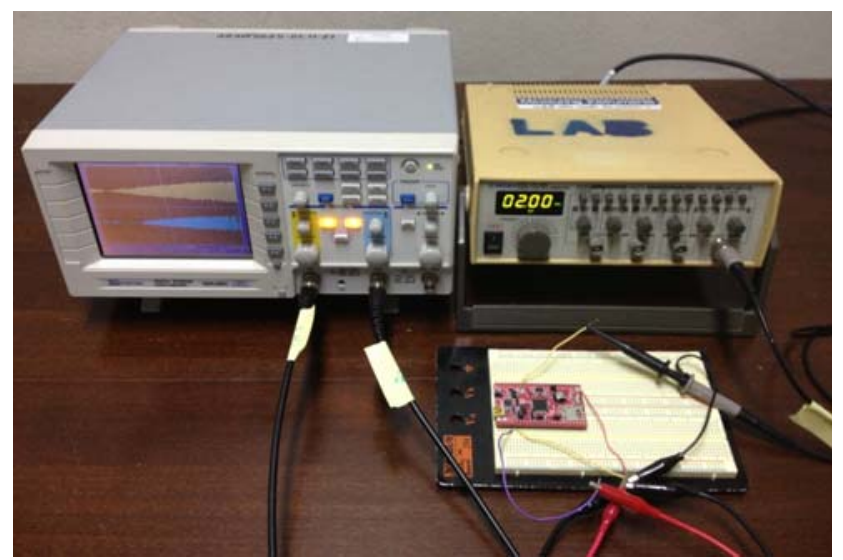

Figure 9 The simulation of the three-way crossover network by using the MATLAB Simulink.

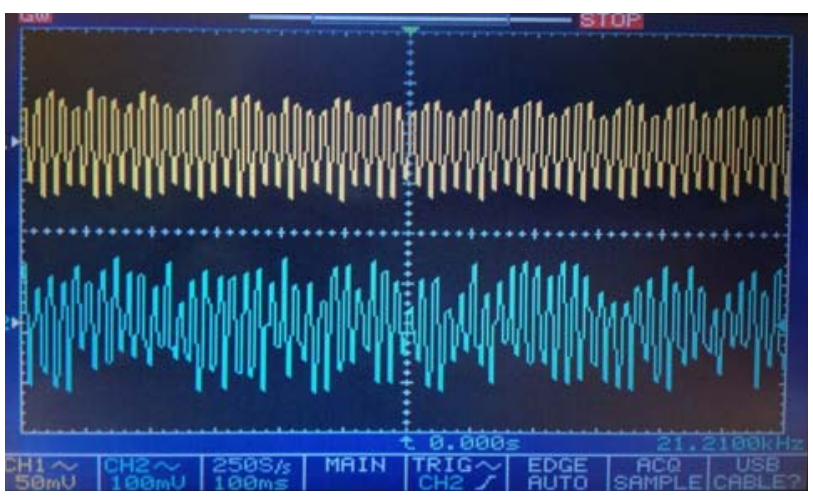

Figure 10. The experimental result of the three-way crossover network via FiO Broad and Rapid32STM Blockset.

\section{REFERENCES}

[1] E. Rapoport, F. A. P. Baruqui, and A Petraglia, "Tunable Analog Loudspeaker Crossover Network," in Proc. The 2003 IEEE Internation Symposium on Circuits and Systems, vol. 5, May, 2003. 
[2] K.Sarangam, A. Sammaiah, and R. K. Jatoth, "Design and Implementation of Universal Filter using Differential Voltage Current Conveyor Suitable for Crossover Networks," in Proc. $2^{\text {nd }}$ IEEE International Conference on Computer Science and Information Technology, Aug 2009, pp.263-267.

[3] Ray Miller, “A Bessel Filter Crossover, and its Relation to Others," RaneNote 147. 2006 Rane Corporation.

[4] Tore A. Nielsen, Loudspeaker Crossover Networks, The Technical University of Denmark: Denmark, 2005.

[5] Henri Korhola, Perceptual Study of Loudspeaker Crossover Filters, Helsinki University of Technology: Finland, 2008.
[6] V. Chutchavong, Linear-Phase Bernstein Filter for Equalized the Distorted Chrominance, LAP LAMBERT Academic Publishing: Germany, 2013.

[7] G. G. Lorentz, Bernstein Polynomials, Chelsea: New York, 1986.

[8] V. Chutchavong, O. Sangaroon, C. Benjangkaprasert, K. Janchipongvej , "Luminance-Chrominance Gain Equalizer Based on Bernstein Polynomials” IEEJ Trans. On Electronics, Information and Systems. Japan, vol. 130, No. 8, pp. 1411-1422, August 2010.

[9] Sanjit K. Mitra, Digital Signal Processing, McGraw-Hill: China, 2001. 